\title{
OTHOLOMTCS
}

Revista de economía, empresa y sociedad

Dosier "Género y empresa: hacia la igualdad real»

Coordinadora: Laura Lamolla Kristiansen

ESTRATEGIAS DE DESARROLLO

\section{Los factores competenciales para crear y hacer crecer la empresa: una perspectiva desde el empoderamiento femenino}

\section{Marta Zaragoza}

Fundadora y directora de Cresàlida y profesora colaboradora de los Estudios de Economía y Empresa de la UOC

RESUMEN Este artículo tiene como objetivo ofrecer algunas estrategias que una mujer puede utilizar para desarrollar su proyecto profesional en el ámbito de la creación y la dirección de empresas, ya sea por cuenta propia o ajena. Asimismo, le permitirá superar las dificultades con las que se encuentre por razón de género a la hora de fijar o lograr sus objetivos.

Para poder comprender los fundamentos de las estrategias y medidas propuestas, en el apartado de la introducción se identifican los principales factores del entorno o extrínsecos que explican la situación que han vivido, y todavía viven, las mujeres de todo el mundo. Esta situación está mayoritariamente caracterizada por la carencia de igualdad de oportunidades en la mayoría de los ámbitos.

El apartado de las estrategias arranca con la que se fundamenta en el modelo de emprendibilidad en clave de competencias y que tiene sus orígenes en el modelo de empleabilidad referenciado en el texto. Este modelo pretende dar respuesta a cuatro preguntas fundamentales que permitan definir y ejecutar el propio proyecto de vida (profesional, empresarial y personal) con mayor garantía de éxito. En cuanto a las otras estrategias, estas se basan en el empoderamiento de la mujer y en el apoyo y el acompañamiento personal y profesional por parte de todas aquellas personas con las cuales pueda establecer una relación de complicidad y confianza.

Finalmente, se propone un modelo de dirección y gestión del equipo humano que ponga el foco de atención en la gestión de la competencia profesional de toda la organización y la igualdad de oportunidades, independientemente de sus factores personales, lo que tiene que permitir construir un equipo con los/las profesionales con quienes se comparte la misión, la visión y los valores del proyecto.

PALABRAS CLAVE emprendeduría; factores competenciales; género. 


\title{
Skills-based factors for creating a company and making it grow: a perspective from the angle of women's empowerment
}

\begin{abstract}
The objective of this article is to offer some strategies which women can use in order to develop their professional plan in the sphere of creating and managing companies, whether they are self-employed or working for someone else. Furthermore, it will allow them to overcome the difficulties that they may encounter due to gender when they set or achieve their objectives.
\end{abstract}

In order to be able to understand the foundations of the proposed strategies and measures, in the introduction section there is an identification of the principal environmental or external factors which explain the situation that women around the world have lived through and continue to live through. This situation is mostly characterised by the lack of equal opportunities.

The section on the strategies starts with that which is based on the entrepreneurship model in skills and which has its origins in the employability model referenced in the text. This model attempts to respond to four fundamental questions which allow for a definition and execution of the life project itself (professional, business and personal) with the greatest guarantee of success. As regards the other strategies, these are based on the empowerment of women, as well as on the support and personal and professional guidance from all those people with whom a relationship of mutual understanding and confidence can be established.

Finally, there is a proposal for a staff management model which places the focus of attention on the management of professional skills around the entire organization, as well as equal opportunities, independent of the personal factors involved, which allows for the building of a team with the male and female professionals who share the mission, vision and values of the project.

KEYWORDS entrepreneurship; skills-based factors; gender.

\section{Introducción: una desigualdad persistente y resistente}

Independientemente de nuestro sexo, todas las personas tendríamos que tener las mismas oportunidades para desarrollarnos personal, profesional y empresarialmente. Por lo tanto, el entorno que tiene que permitir que esta premisa se cumpla tendría que velar por que todas las personas tengamos los mismos derechos, independientemente de nuestros factores personales (sexo, edad, estado de salud, procedencia, etc.).

Hay que remarcar que la experiencia en otros países de Europa confirma que una mayor presencia de mujeres directivas y empresarias conduce a un modelo económico de crecimiento más sostenible. Así pues, está ampliamente estudiado y probado que las organizaciones que tienen mayor cuidado de las personas que trabajan y velan por el bienestar de las personas del entorno en general son más eficientes y eficaces.

Por lo tanto, garantizar la igualdad de oportunidades entre hombres y mujeres conduce a un mundo mejor para todos. Aun así, y según los datos de la Memòria Econòmica de Catalunya del Consell General de Cambres de Catalunya 2018, la presencia de mujeres es claramente inferior a la de los hombres tanto en el ámbito político, empresarial y universitario como en el institucional. Además, la reducción de desigualdades está avanzando muy lentamente (Poveda, 2018). 
A pesar de que se proponen e implementan medidas desde diferentes ámbitos, la desigualdad es persistente y resistente porque, por un lado, su impacto no ha reducido significativamente este sesgo y, por otro, en el ámbito empresarial tampoco se ha conseguido que se implementen de manera generalizada.

Ante un problema que pone también en peligro el crecimiento económico, se han intensificado las medidas en diferentes ámbitos. Desde el ámbito político destacamos la Agenda 2030, aprobada por las Naciones Unidas en septiembre del año 2015, entre cuyos objetivos destacan la reducción de las desigualdades de género y la Ley 17/2015 aprobada por el Parlament de Catalunya que establece medidas transversales en muchos ámbitos de la vida con el fin de erradicar las discriminaciones contra las mujeres.

Aun así, a pesar de que el marco legal sea garante de los derechos fundamentales entre mujeres y hombres, es necesario que también haya cambios de comportamientos y valores por parte de todas las personas, independientemente del rol que ejerzamos: como profesionales, empresarios/as, directivos/as o simplemente ciudadanos/as y consumidores/as, para que el impacto de nuestras decisiones sea positivo en nosotros/as mismos/ as, en las otras personas y en el medio donde vivimos.

Los motivos de las desigualdades están ampliamente estudiados y no son objeto de este artículo, pero es importante no olvidar aquellos que han provocado un mayor y duradero impacto en España. Por un lado, la herencia política franquista que relegó a la mujer a la vida familiar y, por lo tanto, ha alimentado una herencia social machista que continúa viva en muchos ámbitos, como el empresarial. Por otro, el menor valor que se le atribuyen a las actividades en el ámbito familiar y, muy especialmente, a la maternidad respecto al ámbito productivo como consecuencia de un sistema económico extremadamente capitalista.

A todo lo anterior se le añade un entorno político que no ha conseguido crear un sistema que favorezca la conciliación (carencia de infraestructuras sociales, etc.), debido al menosprecio del talento de las mujeres que, precisamente, provocan los primeros motivos apuntados. Este entorno político también ha alimentado (por activa o por pasiva) prácticas empresariales y políticas de gestión del equipo humano que todavía suponen una barrera al desarrollo de las mujeres: incoherencia entre los horarios de las empresas y los de la vida, diferencias en los sueldos por razón de género, diferencias entre los permisos de maternidad y paternidad, etc.

Por último, los medios de comunicación tampoco se han preocupado por visibilizar a las mujeres, entre otros motivos, debido a la connivencia con los poderes económicos y empresariales. Consecuentemente, la menor presencia y visibilidad de mujeres empresarias y directivas ha dejado a las nuevas generaciones huérfanas de referentes que las abran a todas las alternativas laborales y profesionales, perpetuando sectores y profesiones 0 actividades claramente feminizadas o masculinizadas.

Pero para entender los motivos reales de estas desigualdades en el ámbito profesional y empresarial, hay que partir de la idea de que las principales singularidades entre los sexos son debidas a la influencia del entorno y al proceso de socialización al que estamos sometidos/as desde que nacemos. Aunque la propia comunidad científica mantiene un debate abierto y polémico al respeto, este artículo pretende ofrecer una mirada optimista del futuro de la mujer directiva y empresaria, partiendo de las medidas y estrategias que puede diseñar y poner en marcha ella misma. Tal y como se expone más adelante, el entorno influye en la viabilidad del proyecto profesional de cualquier mujer, pero las instituciones representativas de los diferentes entornos (político, educativo, empresarial, etc.) todavía tienen mucho margen para proponer medidas para reducir estas desigualdades y garantizar que cualquier mujer logre sus objetivos. Aun así, en este documento nos hemos querido centrar en una estrategia global y necesaria: el empoderamiento de la mujer para que lidere su propio proyecto de vida, mejore sus posibilidades y disminuya la dependencia de los factores externos.

\section{Impacto de los factores extrínsecos e intrínsecos en el proyecto de vida}

Para analizar la viabilidad de un proyecto profesional, emprendedor o empresarial hace falta centrarse en la persona que lo formula y lo pone en marcha. En este sentido partimos del concepto de empleabilidad. Según Colomer, Palacín y Rubio (2008) es un indicador de la posición que tiene una persona respecto a un empleo concreto en un momento y en un contexto dado. Esta posición viene dada por un conjunto de factores que favorecen o dificultan 
lograr o mantener un objetivo. Concretamente, hablamos de factores del entorno o del contexto y de factores de la persona (personales y competenciales).

Entre los factores del entorno están las decisiones políticas, la coyuntura económica, los hábitos sociales y culturales o las creencias sociales, entre otros. En los factores de la persona intervienen factores personales (las características o circunstancias personales como la edad, el género, el nivel cultural, las creencias propias, la situación económica, trayectoria formativa, profesional, laboral, empresarial, etc.) o competenciales.

Los factores competenciales son factores también de la persona y son el conjunto de conocimientos, habilidades, destrezas y comportamientos laborales. Según el modelo ISFOL adaptado por Colomer, Palacín y Rubio (2008), hay de tres tipos. Las competencias técnicas: los conceptos y las técnicas. Las básicas, el conjunto de conocimientos reconocidos en nuestro contexto sociocultural como requisitos de funcionamiento social; por ejemplo, las competencias digitales y lingüísticas, el cálculo funcional, la lectoescritura, etc. Y, el tercer subgrupo, las competencias transversales, que se ponen en juego en la realización eficiente y eficaz de una actividad laboral o empresarial concreta. Estas últimas son aquellas que aportan más valor porque están presentes en un número más elevado de trabajos y situaciones, por lo tanto, son las más transferibles (por ejemplo, la comunicación, la relación, la iniciativa, etc.).

La metodología de gestión del proyecto profesional fundamentado en la empleabilidad en clave de competencias de Colomer, Palacín y Rubio (2008) promueve que la persona identifique aquellos factores que inciden a favor o en contra de su empleabilidad y tome medidas para reforzar, compensar y/o desarrollar aquello que necesite para lograr y mantener su objetivo.

Este modelo es útil tanto si la persona tiene como objetivo trabajar por cuenta ajena, independientemente del rol que quiera acabar ejerciendo (por ejemplo, como directiva), como si quiere emprender una iniciativa por su cuenta. En este segundo caso, denominamos emprendibilidad a la posición de la persona respecto a su proyecto emprendedor (Zaragoza, 2012).

El concepto de emprendibilidad es igual de válido si la persona tiene una empresa y quiere velar por su continuidad y crecimiento, ya que la emprendibilidad es dinámica. Por lo tanto, es importante evaluar en cada momento de la trayectoria empresarial en qué punto estamos en relación con el objetivo, contrastando entre los factores personales y competenciales que tenemos en aquel momento y los que necesitamos para lograr los objetivos empresariales con el fin de mejorar aquellos aspectos más débiles.

Tenemos que tener muy presente que los factores externos afectarán al proyecto del emprendedor/a o la empresa en función de las circunstancias personales de la persona emprendedora o promotora, pero también en función de su capacidad personal para dar la respuesta más óptima a cada situación; es decir, en función de los conocimientos, las habilidades, las aptitudes y la actitud que tenga a la hora de avanzar con el proyecto. Por este motivo, es básico identificar estos factores externos de forma clara y concreta y diseñar medidas para que no limiten, o lo hagan en menor grado, nuestro proyecto.

En el caso de las mujeres, el talento emprendedor femenino es la expresión resultante de a) la combinación de factores del entorno, o extrínsecos a la mujer, que tienen un determinado grado de incidencia en el proceso y constituyen barreras a su proceso emprendedor por razón de género, y b) de factores personales y competenciales, o intrínsecos a la mujer, que podemos identificar en cuanto a valores, enfoques, conocimientos, habilidades, aptitudes o actitudes, atribuidos mayoritariamente a las mujeres fruto del proceso de socialización (Zaragoza, Torres y Saragossa, 2010).

En general, hay muchos factores extrínsecos que explican las desigualdades entre hombres y mujeres en todos los ámbitos, principalmente en el laboral y empresarial. Consecuentemente, también suponen mayores dificultades para la mujer que quiere crear y consolidar una empresa. Tal y como se ha comprobado en el apartado de la introducción, la mayoría empiezan a afectar desde el propio ámbito familiar a edades tempranas y se retroalimentan en el entorno educativo, primero, y, posteriormente, en el laboral.

Estas barreras sitúan a las mujeres en unas circunstancias personales que hacen peligrar la viabilidad de su proyecto: para poner algunos ejemplos, la menor presencia en ámbitos formales limita las oportunidades de construir una red social y profesional; el rol ejercido dentro de la familia, principalmente como reproductora y responsable de la educación de los hijos, provoca su dependencia económica, entre otros. Esta carencia de poder económico dentro de la estructura familiar se ha traducido en muchos casos en un menor poder de decisión y, 
por tanto, en la imposibilidad de disponer de la inversión para sacar adelante un proyecto empresarial, así como un menor acceso a los sistemas de financiación tradicionales (Zaragoza, 2011).

Pero todos estos factores externos también acaban afectando negativamente a otros factores intrínsecos como la motivación o el propio sistema de creencias, muy influenciado por las creencias del entorno que obstaculizan su proceso y, por lo tanto, la capacidad de sacar adelante iniciativas. En este apartado incluiríamos, por ejemplo, el miedo al fracaso y la conceptualización del éxito o la distancia con el/la referente de empresario/a que tenemos en el imaginario colectivo; este referente se aleja significativamente de los comportamientos y valores que aprecian muchas personas, especialmente las mujeres.

También hemos encontrado muchas situaciones en las que la mujer, después de realizar un análisis comparando los recursos que tendrá que invertir en su trayectoria formativa y profesional (mucho mayores que si fuera un hombre) y los beneficios que obtendrá (mucho menores que si fuera un hombre), acaba abandonando. De hecho, debido al constante menosprecio social de sus capacidades personales y profesionales, muchas mujeres han acabado creyendo que no tienen la capacidad suficiente para ejercer un determinado cargo o posición o realizar ciertas actividades.

Los factores extrínsecos o del entorno solo se pueden cambiar a través de decisiones políticas y/o movimientos sociales, cambios económicos, hábitos, etc. En el caso de los factores personales, algunos no se pueden modificar (por ejemplo, la edad) y otros se mejoran a través de aprendizajes y experiencias, recursos sociales y acciones de asesoramiento.

Pero los factores competenciales son aquellos que realmente se pueden mejorar, todos y cada uno de ellos, a través de la formación, la experiencia profesional o empresarial o la experiencia de vida y, en general, los ponemos en juego en cada situación profesional, empresarial, laboral, familiar, personal, social, etc. En este sentido, es muy importante tener presente que gracias a los factores competenciales podemos reducir el impacto negativo que provocan la mayoría de los factores extrínsecos o externos citados.

Por este motivo, el modelo descrito por Colomer, Rubio y Palacín (2008) hace hincapié en los factores competenciales, porque son recursos que, si se desarrollan, pueden mejorar determinados factores personales y con los cuales se puede evitar que ciertas barreras puedan impedir lograr nuestros objetivos. Precisamente, gracias a que las mujeres han desarrollado unas competencias transversales extraordinarias como consecuencia del proceso de socialización y el rol que históricamente han llevado a cabo en el ámbito social y familiar, han podido lograr los objetivos a pesar de las barreras del entorno.

Algunas de estas competencias, como la comunicación, las relaciones interpersonales, la organización, la negociación, la creatividad e innovación y la gestión de las emociones, entre otras, son capitales para desarrollar una actividad por cuenta ajena, así como para crear y gestionar una empresa. De hecho, las empresas que incorporan mujeres a sus equipos de dirección tienen mejores resultados económicos (Accenture, 2018).

\section{Estrategias para mejorar como empresaria}

\subsection{Dotar el proyecto empresarial de un proyecto de vida}

Además de tener una idea o un modelo de negocio viable técnica y económicamente, hemos constatado la importancia de tener en cuenta de qué manera nos afectarán todos los factores de emprendibilidad. Estos factores intrínsecos y extrínsecos configurarán los pilares de nuestro proyecto de vida y serán la base a partir de la que se construirá la futura iniciativa o continuará creciendo nuestra empresa u organización.

De manera resumida, podemos realizar un análisis cuidadoso de todos estos factores reflexionando en torno a cuatro preguntas fundamentales. ¿Qué quiero? Identificando claramente nuestros intereses y motivaciones. ¿Qué necesito? Es decir, requerimientos del entorno y concretamente identificar qué competencias requiere una persona emprendedora, directiva o empresaria de referencia. ¿Qué tengo? Identificando las propias circunstancias personales (potenciadoras o limitadoras) y las competencias fuertes y a mejorar con relación al perfil emprendedor, directivo o empresario de referencia (Zaragoza y Colomer, 2012). 
En este último apartado de evaluación competencial (¿qué tengo?), hay que recordar que las competencias se adquieren y se mejoran con experiencias formales e informales, poniéndolas en juego en diferentes situaciones (social, profesional, familiar y empresarial) y con diferentes niveles de dificultad. Por lo tanto, es muy importante identificar y tomar conciencia de las competencias que hemos adquirido en los ámbitos informales, con el objetivo de transferirlas al ámbito profesional y empresarial. Esto es especialmente importante para muchas mujeres que no han gestionado nunca una empresa, pero que disponen de competencias transversales para hacerlo, ya que las han adquirido en ámbitos informales.

La cuarta y última pregunta que nos tendremos que hacer es: ¿qué puedo y quiero poner en juego? Contrastando lo qué tengo y lo que necesito, podremos identificar las posibilidades reales de lograr un objetivo y hacer un plan de mejora. Este plan nos servirá para definir medidas para superar las amenazas (externas) o mejorar los puntos más débiles internos, ya sean personales o competenciales.

Para acabar, la coherencia entre los objetivos empresariales, personales y profesionales es básica para garantizar la viabilidad de nuestro proyecto empresarial y, en definitiva, para nuestro proyecto de vida; se trata de confirmar que aquello que queremos en el ámbito empresarial sea coherente con lo que queremos en el profesional y personal, o viceversa.

\subsection{Construir la propia familia adoptiva}

Para hacer frente a todos los factores extrínsecos o del entorno que representan barreras externas al proyecto profesional o empresarial e incluso para adquirir y mejorar las competencias técnicas, básicas y transversales, muchas mujeres echan de menos un entorno emocional de apoyo que les permita mantener o aumentar su motivación, la autoestima y la confianza hacia ella misma y hacia el proyecto. Aun así, muchas veces no solo no lo tienen, sino que este entorno se opone frontalmente.

En estos casos, cuando la familia reconocida socialmente (la que se establece por medio de un vínculo de consanguinidad, matrimonial o cualquier otro vínculo formal) no ofrece a la mujer empresaria o directiva el apoyo que necesita, puede trabajar para construirlo, creando lo que denominamos «familia adoptiva». Es decir, rodeándose de personas que le aporten algún ingrediente que la hagan crecer, que la motiven y la cuiden con el fin de que pueda desarrollar sus proyectos. Más allá de los beneficios técnicos y operativos que necesita, porque esta familia también le permitirá compartir recursos, tecnología, proyectos, clientes, etcétera.

Precisamente, muchas mujeres deciden unirse a redes o asociaciones de mujeres ya existentes por dos motivos: a) porque el hecho de compartir miedos, problemas y necesidades por razón de género genera una confianza de partida que invita a establecer un "contrato emocional» entre los diferentes miembros del grupo desde el principio; b) porque en la cultura empresarial imperante, históricamente, se ha intentado separar la parte personal de la profesional. Por lo tanto, en las instituciones formadas mayoritariamente por hombres, el componente emocional, si bien es implícito porque somos humanos, no se pone de manifiesto explícitamente y se muestran más orientados a los objetivos que a las personas.

Sea como fuere, la experiencia con diferentes tipos de redes evidencia que no siempre lo más óptimo es que el nexo de unión sea el sexo. En muchas ocasiones, la mujer no se sentirá siempre más cómoda con una red de mujeres ni esta cubrirá todas sus necesidades. Esto pasa cuando, aparte de ser mujer y coincidir con algunas de las barreras del entorno, no comparte ni los objetivos ni los intereses, ni siquiera los valores que reinan en aquella red o asociación.

Por ejemplo, formar parte de una asociación de mujeres no es ninguna garantía de que las personas que están defiendan el bienestar común y no actúen por intereses individuales. De hecho, ser mujer tampoco garantiza que se quiera trabajar por un modelo económico y productivo más sostenible en todos los sentidos.

Ante nuevos retos de futuro hay que repensar el trabajo en red para trabajar la igualdad de oportunidades efectiva entre hombres y mujeres, y analizar si las nuevas fórmulas más efectivas pueden implicar contar con aquellos hombres que, conscientes de los efectos y consecuencias de una sociedad que se continúa moviendo por valores y creencias de una cultura machista, comparten con las mujeres una necesidad de cambio, tanto 
dentro de las organizaciones como en el resto de esferas sociales. Cada vez hay más hombres que incorporan a la fórmula del trabajo cooperativo el componente emocional y están dispuestos a establecer un contrato emocional con otros hombres y mujeres con el objetivo de trabajar por el interés común y el bienestar de todo el mundo.

\subsection{Dirigir la organización y gestionar el equipo interno y externo en clave de competencias}

Hombres y mujeres nos hemos socializado de manera diferente y esto nos puede llevar a ejercer distintos estilos de liderazgo. Las diferencias en la socialización hacen que las mujeres tiendan a desarrollar en mayor grado algunas competencias como la comunicación, las relaciones interpersonales y la gestión de las emociones, entre otras. Precisamente, estas competencias contribuyen a lograr una mayor motivación entre las personas que trabajan dentro de una organización y este hecho genera un impacto positivo en toda la actividad (Zaragoza, 2013).

Aun así, la realidad imperante también nos muestra una cara menos amable del liderazgo femenino: cuando una mujer logra un determinado cargo dentro de una organización, en muchas ocasiones acaba adoptando los modelos de gestión tradicionales para conseguir la valoración y el respeto profesional del entorno.

En todo caso, el contexto y sus requerimientos marcan el estilo de liderazgo o el tipo de dirección más óptima para hacer frente a los retos presentes y futuros a los cuales tiene que enfrentarse una organización. En este sentido, los cambios variados y profundos de los diferentes entornos han presionado a las empresas a ser más eficientes y eficaces; a buscar o crear nuevas formas de financiación alternativa; a revisar el modelo de negocio $y$, por lo tanto, han podido identificar nuevos elementos de valor para el mercado; han tenido que innovar, invirtiendo poco o mucho en I+D+l; a abrirse a otros mercados (locales, nacionales e internacionales); a incorporar las tecnologías en muchos de sus ámbitos (producción, comunicación, organización del trabajo, etc.), o a trabajar en red para poder adquirir nueva información y conocimientos, entre otros.

De hecho, todos estos retos comportan poner en juego competencias como la adaptabilidad, la disposición al aprendizaje, la comunicación, la creatividad y la innovación, entre otras muchas competencias transversales, por lo tanto, las personas que están al frente de una organización y toman decisiones que velan por la consolidación y su crecimiento tienen que tener en cuenta que las personas, con su capital competencial, son el principal motor de cambio, de transformación y desarrollo continuo y sostenible tanto de la organización como del territorio. Por lo tanto, tendrían que poner a las personas en el eje central de la actividad de la empresa (Saratxaga, 2009).

La dirección estratégica en clave de competencias es un modelo que, precisamente, permite aprovechar la motivación y las competencias de todas las personas de la organización para tomar decisiones tanto estratégicas como operativas (Zaragoza, 2013). Este principio, a la vez, crea un círculo vicioso muy positivo que se retroalimenta: la mayor participación en las decisiones más importantes de la empresa genera más motivación y esta, a su vez, genera más valor a la empresa. Otro principio de la dirección estratégica en clave de competencias implica facilitar a las personas que alineen su proyecto profesional y personal con la misión, la visión y los valores de la organización a largo plazo.

Precisamente, es un tipo de dirección que beneficia a todo el mundo, pero a las mujeres empresarias en particular. Por un lado, porque pide poner en juego unas competencias que posiblemente habrá tenido la oportunidad de desarrollar en ámbitos informales y podrá transferir a la gestión de la empresa. Por otro, porque contará con el amparo de todo el equipo con todo el apoyo emocional y técnico que supone confiar en todas las personas de una organización.

Finalmente, un último aspecto de este modelo de dirección es gestionar el equipo humano poniendo el foco de atención en el desarrollo de las competencias profesionales y no tanto en las funciones o los objetivos a lograr. El modelo de gestión del equipo humano en clave de competencias permite captar, fidelizar y seguir desarrollando aquellos y aquellas profesionales con el capital competencial que necesitamos en nuestro equipo, y esto implica ajustar muy bien los recursos y las capacidades a la estrategia. Pero lo más importante del modelo es que permite medir estas competencias, principalmente las transversales, superando las dificultades que implica cuantificarlas en comparación con las competencias técnicas y básicas, debido a su mayor grado de subjetividad. 
En definitiva, este modelo permite romper con aquellas dinámicas de selección y promoción interna del equipo humano basadas en factores personales, como son la edad, el género, el lugar de procedencia, el tipo de titulación académica e incluso los rasgos de la personalidad, etc. Estas características personales no aportan ninguna información que, de forma directa, podamos relacionar con lo que las personas saben, saben hacer o quieren hacer y tampoco son buenos predictivos de los comportamientos en un lugar de trabajo o en una actividad. En este caso, las mujeres pueden sacarle el máximo partido a este modelo por el hecho de que sus vivencias, en un entorno que evidencia tantas carencias a la hora de valorar el talento de todas las personas, les pueden aportar una mayor facilidad para comprender los principios en los cuales se basa.

\section{Conclusiones}

Tal y como se ha expuesto en este artículo, históricamente, ha habido un amplio y variado abanico de factores externos que han contribuido, más activa o pasivamente, al menor ritmo en el desarrollo personal, profesional y empresarial de las mujeres, y en demasiadas ocasiones ha significado su freno. De todas maneras, la importancia de velar por la incorporación de las mujeres al mundo laboral como empresarias o trabajadoras en cargos de dirección, con igualdad de oportunidades, es un fenómeno de gran trascendencia en las últimas décadas en todo el mundo por su contribución al desarrollo económico y social, pero, sobre todo, por una cuestión de justicia social.

En cambio, por años que pasen, los datos continúan mostrando diferencias significativas entre hombres y mujeres en la mayoría de los ámbitos y en todo el mundo. Ante esta realidad, en este artículo se ha querido insistir en el hecho de que para salir de este callejón sin salida es imprescindible que la mujer se empodere movilizando sus propios recursos, independientemente de que los factores del entorno o extrínsecos le sean más o menos favorables.

Hemos constatado que las competencias son los recursos más importantes que tenemos para lograr nuestros objetivos y que las adquirimos a través de la formación y la experiencia profesional o empresarial, pero también en la experiencia familiar, personal, etc. Por este motivo, la mujer, debido al rol que ha desarrollado históricamente dentro de la familia como consecuencia de las funciones y las responsabilidades atribuidas por la sociedad por razón de género, ha desarrollado un conjunto de competencias como la comunicación, las relaciones interpersonales, la organización, la negociación, la creatividad y la innovación, entre otras, que puede transferir a cualquier actividad profesional, emprendedora o empresarial con garantías de éxito.

También hemos visto factores personales que tienen una fuerte influencia en la viabilidad de un proyecto profesional o empresarial, como por ejemplo la motivación o las creencias. En aquellos casos en los que la mujer ha identificado con claridad los motivos que la empujan a iniciar un proyecto, le es más fácil desarrollar y mejorar sus competencias y superar determinadas barreras para lograr sus objetivos. Pero en un entorno con tantos estigmas y estereotipos que discriminan a la mujer, hace falta que desarrolle su automotivación y que revise especialmente aquellas creencias que obstaculizan su proceso y, por tanto, la capacidad de sacar adelante iniciativas a pesar de las barreras externas. Principalmente, aquellas que tienen su origen en el sistema de creencias construido socialmente, como por ejemplo el miedo al fracaso y la conceptualización del éxito; estas creencias, cuando se combinan con los estereotipos por razón de género, propician resultados fatales.

Finalmente, es importante valorar la ventana de oportunidad que se abrió durante los años posteriores a la crisis mundial iniciada a finales del 2007. La disminución drástica del bienestar de las personas y de las organizaciones comportó una serie de cambios en los comportamientos y los valores: muchas personas se dieron cuenta de la importancia de que todos y todas trabajemos para que las organizaciones y, por lo tanto, las personas (directivas, empresarias, trabajadoras, emprendedoras, consumidoras, etc.) tengamos una vida más equilibrada y sostenible.

Pero más allá de las buenas intenciones y mejores palabras, hacen falta modelos, metodologías y métodos concretos, como los expuestos en este artículo, que velen por que todo el mundo pueda desarrollarse personal, profesional o empresarialmente, con igualdad efectiva de oportunidades entre mujeres y hombres y, en definitiva, entre todas las personas independientemente de sus factores personales. 


\section{Bibliografía}

ACCENTURE (2018). Getting to Equal [en línea]. https://www.accenture.com/us-en/about/inclusion-diversity/ gender-equality [fecha de consulta: 25/2/2020].

COLOMER, M.; PALACÍN, I.; RUBIO, F. (2008). Claus Tutorials. Guia per a l'acompanyament i el suport tutorial. Barcelona: Fundació Surt/Departament de Treball.

CONSELL GENERAL DE CAMBRES DE CATALUNYA (2018). Memòria Econòmica de Catalunya [en línea]. https:// www.cambrabcn.org/documents/20182/51383/MEC2018.pdf/2fd7f538-c026-4d65-b72d-40dd0b3fa3f1.

POVEDA, C. (2018). Monogràfic. Assolir la Igualtat de Gènere. Objectiu 5 Agenda 2030 [en línea]. http://cads. gencat.cat/web/.content/Documents/Agenda_2030/Jornada_ODS_5/2017-05-26_Agenda-2030_CarmePoveda.pdf.

SARATXAGA, K. (2009). Un nuevo estilo de relaciones. Para el cambio organizacional pendiente. 2a edición. Madrid: Prentice Hall - Financial Times.

ZARAGOZA, M. (2013). Direcció estratègica en clau de competències. Módulo on-line del Programa realizado en el marco de la iniciativa $Q=I$, Accions formatives amb perspectiva de gènere dirigides a les empreses, promovido por la Direcció General de Relacions laborals i qualitat en el treball de la Secretaria d'Ocupació i Relacions Laborals del Departament d'Empresa i Ocupació de la Generalitat de Catalunya.

ZARAGOZA, M. (2013). Estudi sobre el/a perfil professional d'un/a conseller/a d'empresa. Barcelona: Observatori Dona, empresa i economia de la Cambra de Comerç de Barcelona.

ZARAGOZA, M. (2012). Emprendre en clau de competències: el perfil emprenedor. Barcelona: UOC Edicions.

ZARAGOZA, M. (2011). Presència i visibilitat de la dona emprenedora i empresària a la Demarcació de Girona. Departament de Promoció Econòmica i Noves Tecnologies de la Diputació de Girona.

ZARAGOZA, M.; COLOMER, M. (2012). Competències Directives. Barcelona: Mòdul del Postgrau de Gestió de microempreses de la Business School de la Universitat Oberta de Catalunya.

ZARAGOZA, M.; TORRES, E.; SARAGOSSA, A. (2010). Estudi sobre Identificació i Potenciació del Talent Emprenedor Femení. Barcelona: Generalitat de Catalunya. Departament d’Economia i Finances i Surt, Fundació de Dones.

Cita recomendada: ZARAGOZA, Marta. Estrategias de desarrollo. Los factores competenciales para crear y hacer crecer la empresa: una perspectiva desde el empoderamiento femenino. Oikonomics [en línea]. Mayo 2020, no. 13, pp. 1-10. ISSN: 2339-9546. DOI: https://doi.org/10.7238/o. n13.2007 


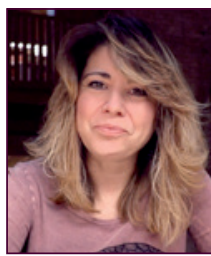

\section{Marta Zaragoza}

mzaragozad@cresalida.com

Fundadora y directora de Cresàlida y profesora colaboradora de los Estudios de economía y Empresa de la UOC.

Economista y emprendedora, especializada en dirección estratégica y la gestión de las competencias profesionales, emprendedoras y directivas en el ámbito del emprendimiento, las organizaciones, la estrategia territorial y la l+D+l. En el 2010 creó Cresàlida con la misión de contribuir a que las organizaciones tengan una vida equilibrada y sostenible a través de una dirección estratégica en clave de competencias.

Ha sido profesora durante más de una década en la Universitat de Barcelona, la Universitat Rovira i Virgili y la UOC en el ámbito del emprendimiento.

Los textos publicados en esta revista están sujetos -si no se indica lo contrario- a una licencia de Reconocimiento 4.0 Internacional de Creative Commons. Puede copiarlos, distribuirlos, comunicarlos públicamente, hacer obras derivadas siempre que reconozca los créditos de las obras (autoría, nombre de la revista, institución editora) de la manera especificada por los autores o por la revista. La licencia completa se puede consultar en https://creativecommons.org/licenses/by/4.0/deed.es_ES.

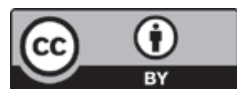

